\title{
Is the Skeptical Attitude the Attitude of a Skeptic?
}

\section{KATARZYNA PAPRZYCKA \\ University of Pittsburgh}

There is something disturbing in the skeptic's claim that we do not know anything. It appears inconsistent because his claim about our not knowing anything seems to contradict his knowing this very fact.

It is noteworthy that it is this point that has been very prominent in various criticisms launched on skepticism. Hegel argues, for example, that the skeptic contradicts himself for there is a deep disparity between his way of life and his self-conception. "[The skeptical consciousness] affirms the nullity of seeing, hearing, etc., yet it is itself seeing, hearing, etc."1 Wittgenstein gives a similar response. He argues that there is a contradiction between the certainty which underlies language-games we engage in (and that we do engage in them is necessary for us to think at all) and the only subsequently expressed doubt. We can doubt something only if we are certain of something else. We cannot doubt everything. "The game of doubting itself presupposes certainty."2

It will be instructive to follow some possible reactions to a skeptical argument. Firstly, it may seem entirely incredible and we may wish to reject it altogether--even if we are unable to find flaws in it. Secondly, we may find it appealing but not bear the consequences the skeptic wants us to bear. Thirdly, of course, we may become convinced and go around launching skeptical arguments ever more refined.

It will be argued here that it is only the second attitude that is genuinely productive and genuinely skeptical, in the sense of being antidogmatic. The first and the last are both dogmatic viewpoints. We may identify the first with the strongly convinced common-sense position (of the Moorean type, for instance), the last--with a thorough-going skeptical stance, and the second--with a "scientific" attitude. If that is correct, as we shall attempt to show in the course of this essay, then the conclusion that suggests itself is that it is the skeptic himself who lacks the skeptical (antidogmatic) attitude, and it is only the attitude of a scientist that is genuinely skeptical.

When speaking of a 'scientist' we do not mean to be reinforcing the ethos of science. The term 'scientific attitude' is supposed to mean 'the (impersonal, "objective") attitude exhibited in, or presupposed by, or

1 G.W.F. Hegel, Phenomenology of Spirit, (Oxford: Oxford University Press, 1979), trans. A.V. Miller, p. 125.

2 L. Wittgenstein, On Certainty, (New York: Harper \& Rows, 1972), \$ 115. 
inherent in, certain scientific procedures.' Here, we shall consider one of them, reduction.

In dealing with the skeptic's contention we will assume a possibilistic ontology. 3 What seems implicit in the skeptic's belief that we may not know anything, is the contention that it is we, of our actual world, who lack the knowledge. And it seems it would not be reasonable for the skeptic to argue that there must be a devil scientist who deceives us by manipulating our brains. The only point that he makes, is that there could be such a scientist. Further, since we do not know whether this is the case or not, we do not have good enough grounds to believe, as we do, that the picture sketched by the skeptic is not indeed true. We can grant then that there is a possible world where people are deceived and thus do not know anything. Let us call it simply the skeptic's world.

Equally, in the world of absolute knowledge people know everything. Between these two extremes there is, of course, a spectrum of possible worlds varying in the degrees of knowledge that our counterparts (and we, among them) possess.

So now the problem of the skeptic appears as that of localization. The question that arises is "Just which of these worlds is ours?". At this point we may paraphrase the skeptic's claim as asserting that our actual world is a skeptic's world. The common-sense dogmatic, on the other hand, believes our actual world not to be a skeptic's world.

The problem of inconsistency arises only at this point. As long as the skeptic does not identify the skeptic's world with our actual world, his talk seems illuminating. "Aha", we may think, "really, we could just as well be deceived". The realm of possibilities becomes open to us, so to speak. But as soon as the inference is made that we know nothing, we should become watchful (as people indeed have been). For the skeptic is just as justified in making this step, as is the common-sense dogmatic. This is also why the best tool in discussion with a skeptic is to ask his question back. For indeed, what reason does the skeptic have for supposing that there is an evil scientist who deceives us? His position is thus on a par with the common-sense one. Either can argue over the other, and the discussion will remain inconclusive. For in all honesty, it seems that we simply do not know which of those possible worlds that vary in deceptiveness, is our actual one.4 And this is the genuinely skeptical (antidogmatic) stance.

3 We shall further assume the framework of David Lewis; cf. On the Plurality of Worlds (Oxford: Blackwell, 1986).

4 The attempts to prove the common-sense position to be better off in probabilistic terms can be just as well made for the skeptical position, cf. Lewis, op. cit., pp. 118-122. 
This, however, is no reason for us to make an ad hoc choice, as it thus has to be. We have to look for a different route. One that seems to suggest itself, is that we ought to take into account all of the possibilities. This indeed seems to be the strategy of the sciences. Let us at first look at one of the mentioned procedures, viz. reduction.

\section{IV}

To keep the consistency of exposition, let us use the possibilistic picture further.

Consider a standard example of reduction, that of Galileo's theory to the theory of gravitation. The theory of free falling bodies has been shown by Newton's theory to have a restricted range of application. It holds approximately when the height from which an object falls is very small in comparison to the radius of the earth. Its success is thus due to the fact that Galileo investigated only a certain subset of phenomena, viz. objects falling close to the surface of the earth, and, of course, due to the fact that the radius of the earth is as long as it actually is.

But surely, if one accepts the possibilistic framework there are possible worlds in which the radius of the earth differs from that of our Earth. Let us consider a subset of these which fulfill the following conditions. First, Galileo's counterparts of these worlds came to the very same ideas as our Galileo did. Second, the theories of the other worldly Galileos were equally well confirmed as that of our actual Galileo. Third, they were confirmed for the very same reasons, viz. that the height from which the considered objects were falling was very small in comparison to the length of the radiuses of the earths.

In those worlds, the other worldly Galileos would have generated the same general formulae except that the gravitational constants would have differed. That is to say, the theories of all these Galileos stand in conflict. Whereas one proposes the gravitational constant to be 9.81 , another claims it to be 10.99 , and so on.

That there is no conflict involved becomes evident only given the gravitation theory. What Newton's theory does is to take into account variables (not considered by Galileo(s)), viz. the mass of the Earth and the distance between its center and the falling body. When these two variables adopt a certain range of values the Newtonian formula yields results similar to those predicted by Galileo. In this sense, the theory of free fall is a special case of Newton's theory.

But, of course, the same will be the case for the other worldly Galileos (given the conditions imposed). Even though the Galilean theories differ from one another and indeed stand in conflict with one another, still each and every one can be reduced to (our) Newton's theory. The latter provides means with which to consider a variety of cases of the length of an earth's radius. In particular, no matter what the length of the radius of the 
earth, that is to say, no matter which of the possible worlds we are talking about, Newton's theory is true. It holds for all those worlds.

The crucial point for us is that by discovering the significance of the new dimensions Newton's theory is able to predict what a Galilean theory would be in each of these possible worlds-given the radius of the carth in that world. This will be the case if there are ways to measure the radius of the earth.

But what if this were impossible? Then we could not know which of the possible worlds we inhabit by merely measuring the radius.

This starts to resemble the situation the skeptic is imagining. We definitely cannot know whether there is a devil scientist who manipulates our thoughts and so we do not know which world we inhabit. Here, we assume that we cannot know which world we inhabit for we cannot measure the radius of the earth.

What then is the scientist to do?

Notice, first, what the skeptic does. He demands that we answer his question, viz. which of the spectrum of worlds is our actual world. His strategy consists in denying us the direct means of doing so and claiming that since we cannot know the opposite (which we are naturally inclined to believe) his conclusion holds. But this, as remarked above, is not the case. What the skeptic manages to achieve, at best, is to put us in state of suspension-we do not know which world we inhabit. In other words, we do not know whether we do or do not know. Or rather, we cannot know whether we do or do not know.

The scientific attitude, on the other hand, as exemplified by reduction, could be paraphrased thus. Let it be granted that we cannot know directly which of the worlds is ours, for example, by not being able to measure the radius of the earth. But instead of jumping to an ad hoc conclusion, let us create a theory which would consider a whole spectrum of worlds differing in the values that the relevant variable adopts and then see whether we can answer the question. And even if we cannot answer it-what have we lost?

In other words, it may be instructive to hold the question in suspension. That does not mean to assume an arbitrary answer, as in the case of the skeptic and the common-sense believer. It is rather to keep all the possibilities in mind and pose other questions taking into account the whole spectrum of possible worlds. In short, why worry about whether there is a scientist who deceives us or not-if we can transcend this by considering situations where he does and where he does not and where he does in varying degrees.

Assuming this strategy, there is nothing to lose and everything to gain. For, as often is the case, the skeptic does not have very much to tell us, except for sowing disbelief. On the other hand, an anti-skeptical attitude is better to the extent that it has something positive to say (although, of course, it may be wrong). Thus, considering a spectrum of which these two 
IS THE SKEPTICAL ATTITUDE THE ATTITUDE OF A SKEPTIC? 123 positions are extremes will tell us at least as much as the collection of these two.

The only remedy of defence is attack, as the old saying goes, it is no wonder that people turn on their heels and go on to do other things. The skeptical doubts should not, however, be neglected altogether, but rather, as the above considerations show, subsumed under more inclusive concepts and conceptions. 